\title{
US diagnosis of acute appendicitis
}

\begin{abstract}
Introduction: Acute appendicitis is the commonest condition requiring acute abdominal surgery. The perforation of the appendix is the most common complication. The fast and accurate diagnosis, as well as the timely surgery could decrease the risk of rupture and minimize the consequences.
\end{abstract}

Aims: The aim of the study was to demonstrate the value of the ultrasound (US) as an excellent diagnostic modality in evaluation of the appendix as well as to assess further treatment of acute appendicitis based on US study.

Methods: We present 97 cases, which were undergone on US examination of the abdomen with special accent on appendicle region. For exploration of the appendix, we used a linear probe from 7, $5 \mathrm{MHz}$.

Results: According of US findings the patients were divided into two groups: I (A) group with the signs of acute appendicitis - 61 cases and II (B) group where the signs of acute appendicitis were absent - 36 cases. From the group I (A) 57 patients were undergone on operative treatment and the US diagnosis of acute appendicitis was confirmed. From the group II (B) 7 patients underwent on operative treatment. We made the comparison between the US findings, operative and histology findings and our results correlated with the results from the literature data.

Conclusion: US are an extremely valuable diagnostic method in evaluation of the appendix with high diagnostic accuracy especially in the presence of acute inflammatory changes, with possibilities to plan further operative treatment.
Volume 5 Issue 3 - 2018

\section{Antonio Gligorievski}

University Clinic of Surgery St. Naum Ohridski Skopje, Macedonia

Correspondence: Antonio Gligorievski, University Clinic of Surgery St. Naum Ohridski Skopje, Macedonia ul.Vasil Gjorgov br. 28/25, 1000, Skopje, Macedonia, Email atglmed@gmail.com

Received: May 10, 2018 | Published: June 22, 2018

Keywords: appendix, uncompressible appendix, acute appendicitis, US

\section{Introduction}

Acute appendicitis presents as an acute inflammation of the appendix and it is the commonest condition requiring acute abdominal surgery. ${ }^{1}$ About $7 \%$ from the population will have inflammation of the appendix during their lifetime, and the peak incidence is in the second and third decades of life., ${ }^{2,3}$

The classic symptoms of the acute appendicitis for the first time were described by Fitz in 1886. According the histology there are different stages of the acute appendicitis as follows: early appendicitis (appendicitis acuta recens); appendiceal phlegmon (appendicitis suppurativa acuta), gangrenous appendicitis (appendicitis acuta gangraenosa) with perforation that follows.

Anorexia, nausea, vomiting and pain are the commonest symptoms related to the acute appendicitis. The pain begins in the epigastria, gradually moves to the periumbilical region and finally over a period of 1-12 hours, localizes in the right lower quadrant in more than 50\% of the patients i.e. with sensitivity and specificity of $80 \%$. The pain becomes diffuse in peritonitis with the direct or rebound tenderness of the abdominal muscles. The duration of the symptoms longer than 36 hours is unusual in unperforated appendix.

The obstruction of the lumen of the appendix is the principal cause for distension of appendix due to accumulation of intraluminal fluid. The obstruction has multiple causes, including lymphoid follicular hyperplasia, fechaliths, appendicoliths, parasites, foreign bodies, Crohn's disease, primary or metastatic cancer, and carcinoid and cecum cancer.
The lymph and venous drainage allow bacterial invasion into the bowel wall and at the late stages perforation and penetration of the pus into the peritoneal cavity.

The diagnosis of the appendix is based on history, physical examination, laboratory findings, but one third of patients with acute appendicitis have atypical history and physical examination. In this group of patients the radiological methods have important clinical role.

Puylaert ${ }^{1}$ described technique for exploration of the appendix with dosed compression over the appendix using transabdominal transducer and he established the criteria that allow standardization of the ultrasound (US) study of the appendix. The criteria for the inflammation of the appendix are as follows: outer diameters that exceed $6 \mathrm{~mm}$; uncompressible appendix; absence of peristalsis; presence of the periappendicular fluid; appendicoliths; periappendicular infiltrate. The normal appendix cannot be visualized, but in most of the cases the inflammated one can be.

The perforation of the appendix is the most common complication. The fast and accurate diagnosis, as well as the timely surgery could decrease the risk of rupture and minimize the consequences. ${ }^{4}$

With use of US and CT and with use of the established criteria the timely and accurate diagnosis is made up with reduction of the number of the unnecessary surgery and the number of appendiceal perforation as well. ${ }^{5-8}$ 


\section{Aim}

To demonstrate the value of the ultrasound (US) as an excellent diagnostic modality in evaluation of the appendix as well as to assess further treatment of acute appendicitis based on US study. The use of US in diagnosis of the acute appendicitis has an objective to decrease the number of perforation as a result of the late diagnosis as well as the number of unnecessary laparotomy as a consequence of false positive history and physical examination.

If the cause of pain in right lower abdominal quadrant according to the US findings is the inflammation of the appendix to suggest further treatment.

\section{Material and methods}

We show the cases performed as an emergency cases at the Institute of radiology in cooperation with the Clinic for Abdominal Surgery in Skopje in duration of sixth month period.

We demonstrate 97 cases with history and physical examination of acute appendicitis, aged 15-57, with peak incidence in second decade of life, $36(37,1 \%)$ are male and $61(62,9 \%)$ are female (Table 1). For the exploration of the appendix we used ultrasound apparatus Toshiba sonolayer SL-250 so linear transducer of $7,5 \mathrm{MHz}$, with dosed compression. The dosed compression makes the air go away from the bowel loops and decreases the distance among the transducer and the appendix, with what we have better visualization of the appendix. The careful exploration of the ileocecal region is performed, and the leading points are: umbilicus, inguinal ligament Pupartty, ileopsoas muscle, cecum and the iliac artery and vein. After each exploration of the appendiceal region we performed examination of whole abdomen using $3,75 \mathrm{MHz}$ transducer.

Table I According to the gender and age of patients

\begin{tabular}{lllllll}
\hline Age & II-20 y. & 2I-30 y. & 3I-40 y. & 4I-50 y. & 5I-60y. & Total \\
\hline Male & 19 & II & 3 & 2 & I & 36 \\
Female & 36 & 16 & 5 & 3 & I & 61 \\
Total & 55 & 27 & 8 & 5 & 2 & 97 \\
\hline
\end{tabular}

Dominant age is between II and 20 years with 55 cases (56.7\%) and females with 36 cases $(37,1 \%)$.

\section{Results}

The classic history of acute appendicitis is: pain in the right lower quadrant in all patients, nausea, vomiting and anorexia in most of the patients, slightly elevated temperature in 46 patients, peritoneal sign, localized tenderness on percussion in 12 patients; positive Rovsing sign, pain at the right lower quadrant during palpation, pain at the left lower quadrant in 23 patients, psoas sign during extension of the right thigh in 5 patients, obturator sign during internal rotation of the flexed right thigh in 3 patients and Dunfi sign of pain at the right lower quadrant in 7 patients. The duration of the symptoms is lesser then 48 hours in $80 \%$, but it can be longer in elderly with appendiceal perforation.

From laboratory studies characteristic is moderate leukocytosis in $74 \%$ of patients from 10000 to 14000 , and $26 \%$ had normal white blood cell count.

The basic US criteria for the diagnosis of acute appendicitis that we use are as follows: visualization of distended appendix fluid filled, appendix with diameter that exceed $6 \mathrm{~mm}$; appendiceal wall thicker then $3 \mathrm{~mm}$; absence of peristalsis and uncompressible appendix; forming of infiltrate around the appendix and free fluid into the appendiceal surrounding or into the abdomen.

In patients that we did not visualize the appendix, based on the US criteria there is no presence of acute appendicitis; and it is possible to obtain false negative results.

The inflammated appendix is visualized medial and inferior from the cecum, sausage-like, blind ending structure at the longitudinal plane or target sign (bull's eye appearance) at axial plane.

The appendix on US study is demonstrated by: hypo echoic lumen, hyper echoic mucosa, iso echoic lamina propria, hyper echoic submucosa, hypo echoic muskularis and hyper echoic serosa. The increased size of the appendix is a sign for phlegmonous or gangrenous appendicitis (Figure 1). The present appendicolith is demonstrated as hyper echoic mass that fills in the lumen and gives acoustic shadow (Figure 2B). When the appendicolith should be visualized, the wall thickness and the compressibility are not the features for making the diagnosis of acute appendicitis. The gangrenous appendicitis has changes in echogenity in all layers and the same are with the uniform echogenity and cannot be distinguished (Figure 3A). If the ill defined or hypo echoic mass is seen that surrounds the appendix that presents the periappendiceal inflammation (Figure 4). The ill defined appendiceal wall is suggestible, but not diagnostic for the periappendiceal process. The fluid presence into the appendiceal surrounding, with appendiceal wall echogenity changes is US feature for perforation (Figure 3B). The positive lymph nodes are oval hypo echoic and they don't change the shape on compression.
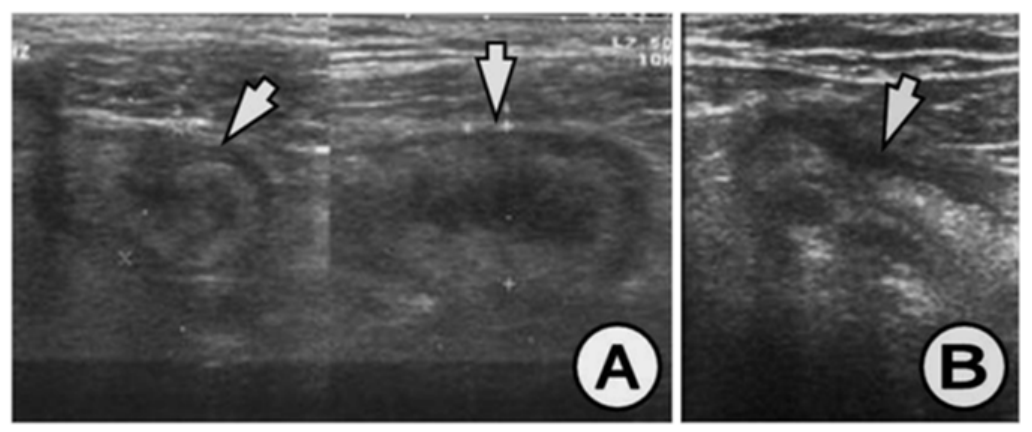

Figure I (A) Appendix increased in size, $17 \mathrm{~mm}$ in diameter, thickened wall greater than $6 \mathrm{~mm}$, (B) Appendix with widened lumen with dense contents, with 18 $\mathrm{mm}$ in diameter and thickened wall greater than $5 \mathrm{~mm}$. US finding for appendiceal phlegmon. 

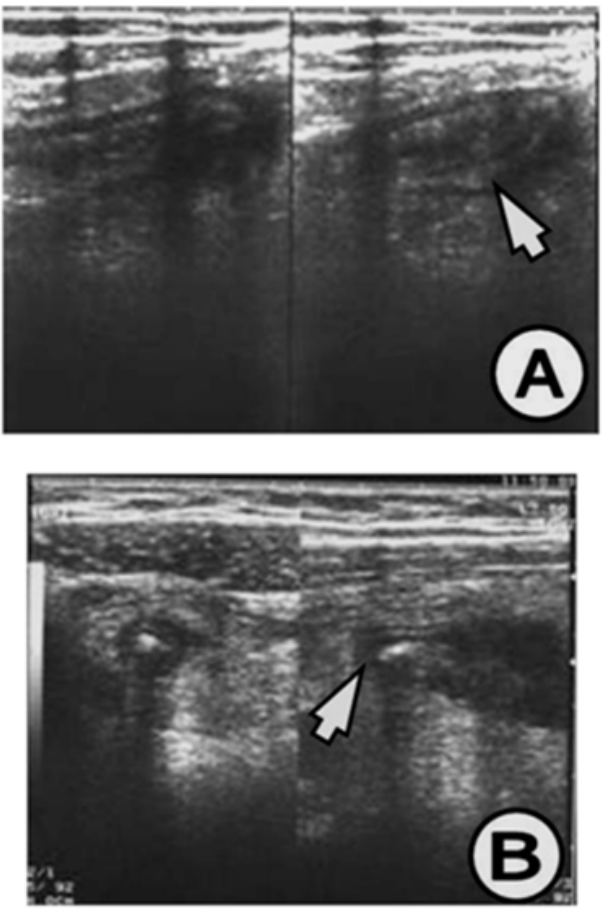

Figure 2 (A) Appendix increased in size, thickened wall, widened lumen, US finding for appendiceal phlegmon, (B) Clearly visualized appendicolith into the lumen of the appendix, which is with thickened wall and widened lumen, US finding for appendiceal phlegmon.

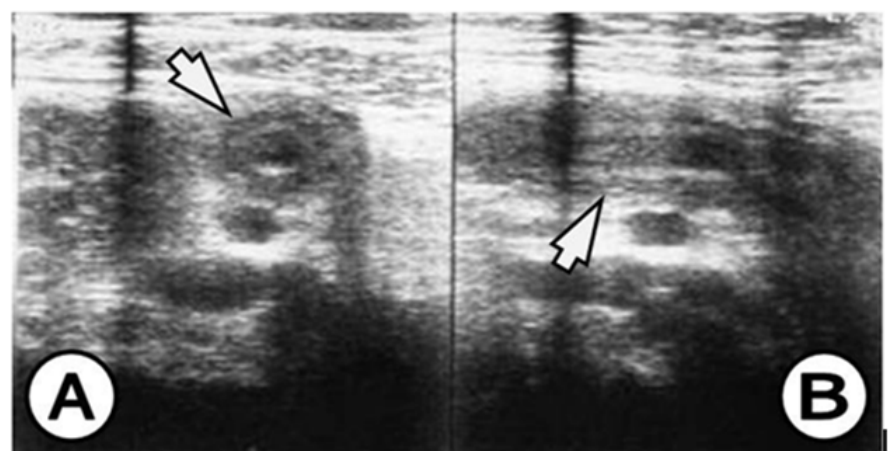

Figure 4 (A) Coronal view, appendix with thickened wall and surrounding infiltration, (B) Saggital view, appendix with thickened wall and surrounding infiltration, US finding for appendiceal phlegmon.

The patients based on the US findings are divided into two groups: I (A) group with the signs of acute appendicitis - 61 cases and II (B) group where the signs of acute appendicitis were absent -36 cases (Table 2). From the group I (A) 57 patients were undergone on operative treatment and the US diagnosis of acute appendicitis was confirmed. From the group II (B) 7 patients underwent on operative treatment.

In terms of the size of the appendix we divided the patients into two groups: group with the diameter up to $12 \mathrm{~mm}$ where we have 39 patients and group with diameter greater then $12 \mathrm{~mm}$ in size where we have 22 patients, from which 7 patients were with diameter greater than $17 \mathrm{~mm}$. In terms of the appendiceal wall thickness in 43 cases it was between 3 to $5 \mathrm{~mm}$, and in 18 it was greater than $5 \mathrm{~mm}$. In 13 patients we detected appendicoliths into the lumen of the appendix.
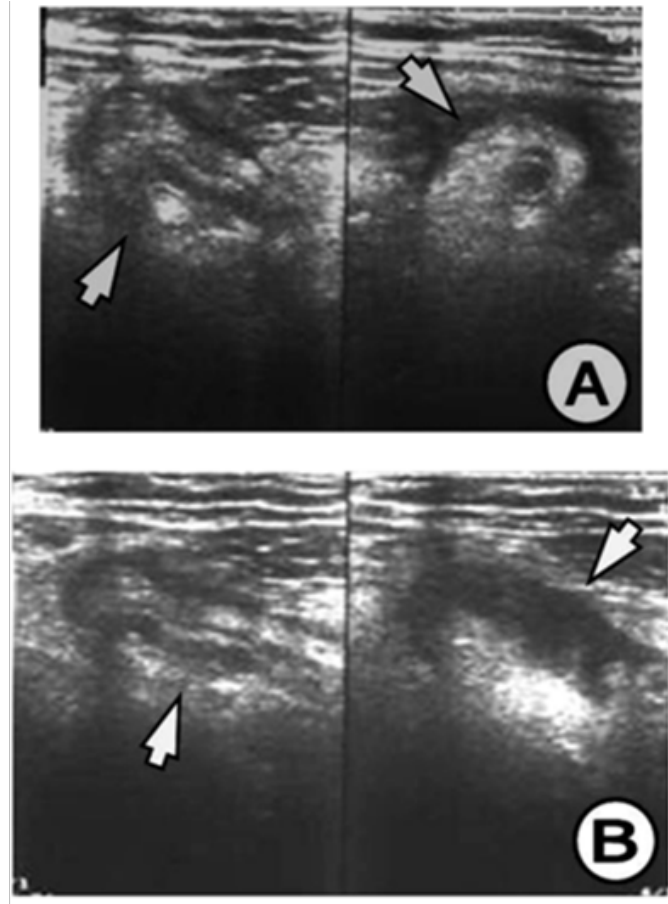

Figure 3 (A) Appendix in saggital and coronal plane, thicken and oedematous wall, with widen lumen, disturbed appendiceal architecture and surrounding infiltration, US finding for gangrenous appendicitis, (B) Appendix with thickened wall, widened lumen and periappendiceal fluid, US finding for gangrenous appendicitis.

Table 2 According to the US findings and surgery findings

\begin{tabular}{lll}
\hline & US findings & Surgery findings \\
\hline I (A) group (6I cases) & Positive in 6I & Positive in 57 \\
II (B) group (36 cases) & Negative in 36 & Positive in 7
\end{tabular}

According to the US findings the patients were divided into two groups: I (A) group with US findings for acute appendicitis in 61 cases, surgery confirms 57 cases and II (B) group with negative US findings for acute appendicitis in 36 cases, which from 7 cases were false negative.

In all cases obstruction of the appendiceal lumen was caused by follicular hyperplasia, bowel contents, appendicolithes, except in one case where obstruction was caused by cancer of the cecum.

Based on the US finding we have 14 patients with early appendicitis (Figure 5), 31 patients with appendiceal phlegmon (Figure 2A) and 12 with gangrenous appendicitis (Figure $3 \mathrm{~A}$ ) from which 4 cases are with appendiceal perforation (Figure 6).

The group of patients that are with no remarkable signs for the presence of acute appendicitis, we have 7 cases with mesenterial lymphadenitis, from which 2 patients had clear US finding of acute appendicitis one month thereafter.

The correlation is done among the preoperative US diagnosis and the operative and histology findings with the accuracy: for the early acute appendicitis is $11 / 14$, for the suppurative appendicitis is $25 / 31$, for gangrenous appendicitis is $7 / 8$ and for perforated appendicitis is $4 / 4$, or in total $47 / 57(82,45 \%)$ (Table 3$)$. 
Table 3 According to the US findings in correlation with histology findings

\begin{tabular}{llllll}
\hline \multirow{2}{*}{ US findings } & \multicolumn{5}{l}{ Histology findings } \\
\cline { 2 - 6 } & EA & APh & GA & PA & Total \\
\hline EA & II & 3 & & & 14 \\
APh & 2 & 25 & 4 & & 31 \\
GA & & 1 & 7 & & 8 \\
PA & & & & 4 & 4 \\
Total & 13 & 29 & II & 4 & 57 \\
\hline
\end{tabular}

Abbreviations: EA, early appendicitis; APh, appendiceal phlegmon; GA, gangrenous appendicitis; PA, perforated appendicitis.
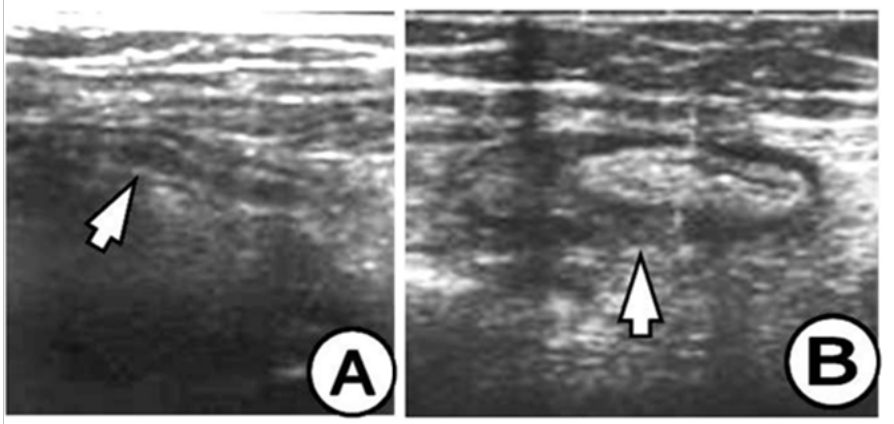

Figure 5 (A) Clearly visualized appendix, with diameter of $8,7 \mathrm{~mm}$ and thickened wall of $3,5 \mathrm{~mm}$, US finding for early appendicitis, (B) Thicken wall of the appendix and widen lumen, US finding for early appendicitis.

\section{Discussion}

Acute appendicitis remains one of the most common surgical emergencies with a lifetime occurrence of $7 \% .{ }^{9,10}$ We could tell that with the use of US in the diagnosis of the acute appendicitis there is decreasing of the unnecessary surgery, decreasing the number of the false negative findings and postponing the surgery only based on the history and physical examination. Early ultrasound detection and surgical intervention is the best way to manage acute appendicitis in order to reduce complications and mortality. ${ }^{10-13}$ Lee et al., ${ }^{10}$ showed that the incidence of acute appendicitis increased in patients younger than 10 years and older than 50 years. The highest rate of acute appendicitis in our sample was found in the age group 11-20 years.

More publications suggest that the US has great diagnostic value in acute appendicitis. The prospective studies showed that in $18 \%$ to $26 \%$ of patients final diagnosis could be established with US and should undergo on surgery. ${ }^{1}$ With this method the number of the negative appendectomies is reduced on 8,5\% do $13,2 \% .{ }^{1}$ Skaane et al. ${ }^{5}$ Suggest high correlation among the operative and histology finding, and the sensitivity of the US method into the diagnosis of the inflammated appendix is $78,92 \%$, and the specificity is 87 Dreuw et al., ${ }^{4}$ in their study the US finding compare with the clinical diagnosis and the histology finding. For the appendiceal region mass the sensitivity is $100 \%$ and for appendiceal phelgmon is $88 \%$. The specificity of the method is $97 \%$, positive predictive value is $97 \%$, and the negative predictive value is $47 \%$. The US finding correlates with the operative finding in $87 \%{ }^{4}$
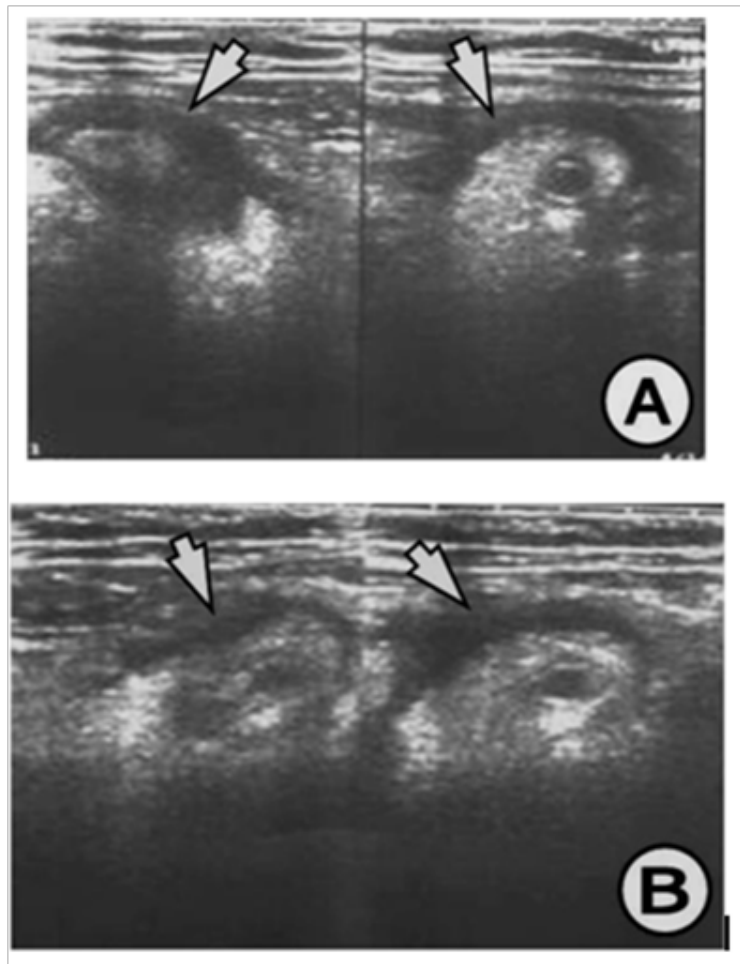

Figure 6 (A) US of acute appendicitis. (B) Appendix with widened lumen and thickened wall, disturbed echogenity, periappendiceal fluid, US finding for perforated gangrenous appendicitis.

In most of the cases that valorize the contribution of US into the diagnosis of the acute appendicitis the sensitivity is from $75-90 \%$, and specificity is from $86-100 \%$ and the diagnostic accuracy is from 87 $96 \%$. $13,6,7,14,15$

Most of the newly publications demonstrated that the greatest diameter of the appendix is $>6 \mathrm{~mm}$, there is about inflammation in $93 \%$ of the cases.

According to Lane et al., ${ }^{8}$ the sensitivity of the $\mathrm{CT}$ in diagnosis of the acute appendicitis is $90 \%$, specificity is $97 \%$, while the new publication demonstrate sensitivity of $96 \%$ and specificity of $98-99 \%$.

According to Balthazar et al., ${ }^{16}$ who analyzed the data from CT and US the sensitivity is $96 \%$ over $76 \%$, specificity is $89 \%$ over $91 \%$, accuracy is $94 \%$ over $83 \%$, PPV is $96 \%$ over $95 \%$ and NPV is $95 \%$ over $76 \%$.

Al-Ajerami ${ }^{17}$ report the overall specificity and sensitivity of $84.8 \%$ and $83.3 \%$ respectively, which showed that ultrasound has a relatively high specificity and sensitivity in diagnosing appendicitis especially with patients of normal weight. Our results compare well with other studies reported sensitivity $75 \%-98 \%$, specificity $86 \%-100 \%$ with positive and negative predictive values of $91 \%-100 \%$ and $89 \%-$ $99 \%$. $^{18-20}$

The barium enema in diagnosis of the appendix is with sensitivity of $80-100 \%$. Its advantage is that the examination is simple with using simple materials and allows to be diagnosed other diseases such as Crohn's disease, colon cancer, ischemic colitis that might simulate 
appendicitis. The disadvantages are: radiation, non diagnostic examination, insufficient examination and invasive examination. Due to all these disadvantages the barium enema is not adequate as a screening method for the diagnosis of appendicitis, especially in the era of US. In spite of the diagnostic value of the barium enema study for appendicitis it is not used as screening method.

In most of the cases the appendix has intraperitoneal location, and it could contact the parietal peritoneum when it is inflamed. In $30 \%$ of the cases the appendix could be hidden from the anterior peritoneum due to its pelvic or retro colic location. ${ }^{6}$ The poster lateral approach is better for the exploration of the retrocecal region.

The differential diagnosis includes: endometriosis, ovarian cyst or torsion, tubo-ovarial abscess, degenerative fibroids of the uterus, diverticulitis, Crohn's disease, colon cancer, cholecistitis, bacterial enteritis, mesenterial adenitis and torsion of the omentum.

The number of the negative appendectomies varied from $16,7 \%$ to $47,2 \%$, with mean of $26,4 \% .^{2}$ The false negative diagnosis are explained by perforation of the inflamed appendix from $9,6 \%$ to $32,2 \%$, with mean of $22,6 \% .^{2}$ Ultrasound may not detect the appendix when it is retrocaecally located or if it lies deep in the pelvis. ${ }^{21-23}$ Indications for $\mathrm{CT}$ after sonography included retrocecal appendicitis, nonvisualization of the appendix by sonography, an equivocal findings with secondary signs of appendicitis, and negative findings with clinical concern for appendicitis. ${ }^{22,23}$ The negative appendectomy rate can be significantly reduced by using ultrasound coupled with clinical assessment and evaluation. ${ }^{24-26}$

\section{Conclusion}

We can conclude that the US has great diagnostic value in acute appendicitis. The advantage consists of: easy performance, noninvasive method, no radiation, short examination duration, the possibility for detection of other causes of distal abdominal pain, especially in pregnant women. The disadvantage is that the finding depends on the operator. The non visualized appendix is interpreted as non inflamed appendix, so it is from great importance the examination to be performed by experienced radiologist with attention and he would be able to accomplish maximal sensitivity.

If the examination with the dosed compression of the right lower quadrant is positive for the appendicitis, the surgery is recommended. If the finding is unremarkable, it is not enough to exclude appendicitis. In that case if the pain and the history are still present the follow up US is recommended for further observation by the abdominal surgeon.

With use of US and with use of exact established standards and criteria the timely and accurate diagnosis of acute appendicitis is allowed. It will reduce the number of the perforations and unnecessary surgery.

\section{Conflicts of interest}

Dr. Antonio Gligorievski declares that there is no conflict of interest.

\section{Funding}

There are no sponsors for this article.

\section{Ethical approval}

Ethical approval was not necessary for this article.

\section{Consent}

Written informed consent was obtained from the patient for publication of this case report and accompanying images. A copy of the written informed consent is available for review by the Editor in Chief of this journal on request.

\section{References}

1. Puylaert JB. Acute appendicitis US evaluation using graded compression. Radiology. 1986;158(2):355-360

2. Puylaert JB. The use of ultrasound in patients with clinical signs of appendicitis. Thesis, Netherlands, Drukkerij de Kempaenaer Oegstgeest: University of Leiden; 1988.

3. Jeffrey RB Jr, Laing FC, Lewis FR. Acute appendicitis: high resolution real time US findings. Radiology. 1987;163(1):11-14.

4. Dreuw B, Truong S, Reisner KP, et al. The value of sonography in the diagnosis of appendicitis. A prospective study of 100 patients. Chirurg. 1990;61(12):880-886.

5. Skaane P, Amaland PF, Nordshaus T, et al. Ultrasonography in patients with suspected acute appendicitis: a prospective study. $\mathrm{Br} J$ Radiol. 1990;63(754):787-793.

6. Worrell JA, Droshagen LF, Kelly TC, et al. Graded compresion ultrasound in the diagnosis of appendicitis. A comparison of diagnostic criteria. $J$ Ultrasound Med. 1990;9(3):145-150.

7. Garcia-Aguayo FJ, Gil P. Sonography in acute appendicitis: diagnostic utility and influence upon management and outcome. Eur Radiol. 2000;10(12):1886-1893.

8. Lane MJ, Liu DM, Huynh MD, et al. Suspected acute appendicitis: nonenhanced helical $\mathrm{CT}$ in 300 consecutive patients. Radiology. 1999;213(2):341-346.

9. Styrud J, Josephson T, Eriksson S. Reducing negative appendectomy: evaluation of ultrasonography and computed tomography in acute appendicitis. Int J Qual Health Care. 2000;12(1):65-68.

10. Lee SL, Walsh AJ, Ho HS. Computed tomography and ultrasonography do not improve and may delay the diagnosis and treatment of acute appendicitis. Arch Surg. 2001;136(5):556-562.

11. Doria AS, Moineddin R, Kellenberger CJ, et al. US or CT for diagnosis of appendicitis in children and adults? A meta-analysis. Radiology. 2006;241(1):83-94.

12. Mostbeck G, Adam EJ, Nielsen MB, et al. How to diagnose acute appendicitis: ultrasound first. Insights Imaging. 2016;7(2):255-263.

13. Hernandez JA, Swischuk LE, Angel CA, et al. Imaging of acute appendicitis: US as the primary imaging modality. Pediatr Radiol. 2005;35(4):392-395.

14. Schwerk WB, Wichtrup B, Rothmund M, et al. Ultrasonography in the diagnosis of acute appendicitis: a prospective study. Gastroenterology. 1989;97(3):630-639.

15. Wu J, Zhang T, Zhu Y, et al. Diagnostic value of ultrasound compared to CT in patients with suspected acute appendicitis. Int J Clin Exp Med. 2017;10(10):14377-14385 
16. Balthazar EJ, Birnbaum BA, Yee J, et al. Acute appendicitis: CT and US correlation in 100 patients. Radiology. 1994;190(1):31-35

17. Al-Ajerami Y. Sensitivity and specificity of ultrasound in the diagnosis of acute appendicitis. East Mediterr Health J. 2012;18(1):66-69.

18. Ayaz A, Lubna MV, Tanvir K, et al. Diagnostic accuracy of alvarado score in the diagnosis of acute appendicitis. Pakistan Journal of Medical Sciences. 2009;25(1):118-121.

19. Paulson EK, Kalady MF, Pappas TN. Clinical practice. Suspected appendicitis. N Engl J Med. 2003;348(3):236-242.

20. Summa M, Perrone F, Priora F, et al. Integrated clinical-ultrasonographic diagnosis in acute appendicitis. J Ultrasound. 2007;10(4):175-178.

21. Gracey D, McClure MJ. The impact of ultrasound in suspected acute appendicitis. Clin Radiol. 2007;62(6):573-578.
22. van Breda Vriesman AC, Puylaert JB. Mimics of appendicitis: alternative nonsurgical diagnoses with sonography and CT. AJR Am J Roentgenol. 2006;186(4):1103-1112.

23. Johansson EP, Rydh A, Riklund KA. Ultrasound, computed tomography, and laboratory findings in the diagnosis of appendicitis. Acta Radiol. 2007;48(3):267-273.

24. Assefa G, Meseret S, Nigussie Y. The role of ultrasound in diagnosing acute appendicictis. Ethiop Med J. 2006;44(1):67-74.

25. Chang YJ, Kong MS, Hsia SH, et al. Usefulness of ultrasonography in acute appendicitis in early childhood. $J$ Pediatr Gastroenterol Nutr. 2007;44(5):592-595.

26. Lee SL, Ho HS. Acute appendicitis: is there a difference between children and adults? Am Surg. 2006;72(5):409-413. 